Cite this: RSC $A d v$., 2013, 3, 26451

\title{
A microfluidic system supports single mouse embryo culture leading to full-term development $\uparrow$
}

\author{
Telma Cristina Esteves, ${ }^{a}$ Fleur van Rossem, ${ }^{\text {b }}$ Verena Nordhoff, ${ }^{\mathrm{c}}$ Stefan Schlatt, ${ }^{\mathrm{c}}$ \\ Michele Boiani ${ }^{a}$ and Séverine Le Gac ${ }^{\star b}$
}

\begin{abstract}
The present study demonstrates the feasibility of application of a microfluidic system for in vitro culture of preimplantation mouse embryos, with subsequent development to full-term upon embryo transfer. Specifically, embryos cultured in groups in $\mathrm{nL}$ volume chambers achieve pre-implantation developmental rates up to $95 \%$ (4.5 days after fertilization), while birth rates upon transfer in utero are comparable to conventional droplet culture ( 30\%). Importantly, while culturing single embryos in conventional microliter droplets hampers fullterm development, mouse embryos cultured individually in a confined microfluidic environment achieve normal birth rates (29-33\%) with normal morphology. Furthermore, the refreshment of culture media (dynamic culture) during pre-implantation in the microfluidic system does not impair development to term. These results deliver great promise to studies in developmental biology and human assisted reproductive technologies (ART), as nanoliter culture volumes provided by microfluidics will (1) allow online screening of physical and chemical culture parameters and (2) facilitate the acquisition of physiological data at the single embryo level - essential requisites for the determination of optimal embryo culture conditions.
\end{abstract}

Received 18th August 2013 Accepted 14th October 2013

DOI: 10.1039/c3ra44453h

www.rsc.org/advances
Here, we propose that microfluidics can contribute to this decisive issue of the field of ART by offering a customized microenvironment that resembles that found in the oviduct, as well as integrated platforms with parallelization potential for single embryo manipulation with direct feedback on their development. First, the micrometer-sized dimensions found in microdevices lend themselves well to fine-tuning of the physical and chemical microenvironment of the culture, ${ }^{6}$ to the creation of a dynamic environment as well as spatial and temporal gradients, ${ }^{7}$ as found in vivo. Also, this format is ideal for cell experimentation down to the single cell level, ${ }^{8}$ and has proven its suitability for studies on embryos of various species, down to the single embryo level. ${ }^{9-11}$ Finally, microfluidic constructs can easily be multiplexed and include miniaturized sensors to monitor in situ specific physical or (bio)chemical parameters. So far, despite its tremendous potential, microfluidics has not yet been appropriately explored for embryo culture: embryos have only been cultured in groups in microfluidic devices, ${ }^{12-14}$ in unconfined volumes of medium, ${ }^{15-17}$ and attempts to realize (semi)dynamic culture ${ }^{12,18}$ has resulted in embryo viability loss.

Here, we present the first-ever study in which microfluidic and droplet culture system are compared side by side for their ability to support the entire development of mouse embryos, using embryo densities down to one (as is the case in human ART). Various culture conditions are screened and their impact on the embryo development is assessed, including full-term development (Fig. 1). Specifically, mouse embryos are cultured throughout their preimplantation development, from the zygote stage until 3.5-4.5 days post coitum (dpc) in both $\mathrm{nL}$ chambers (30 or $270 \mathrm{~nL}$ ) and conventional drops, individually or as groups (5 or 20). In one set 


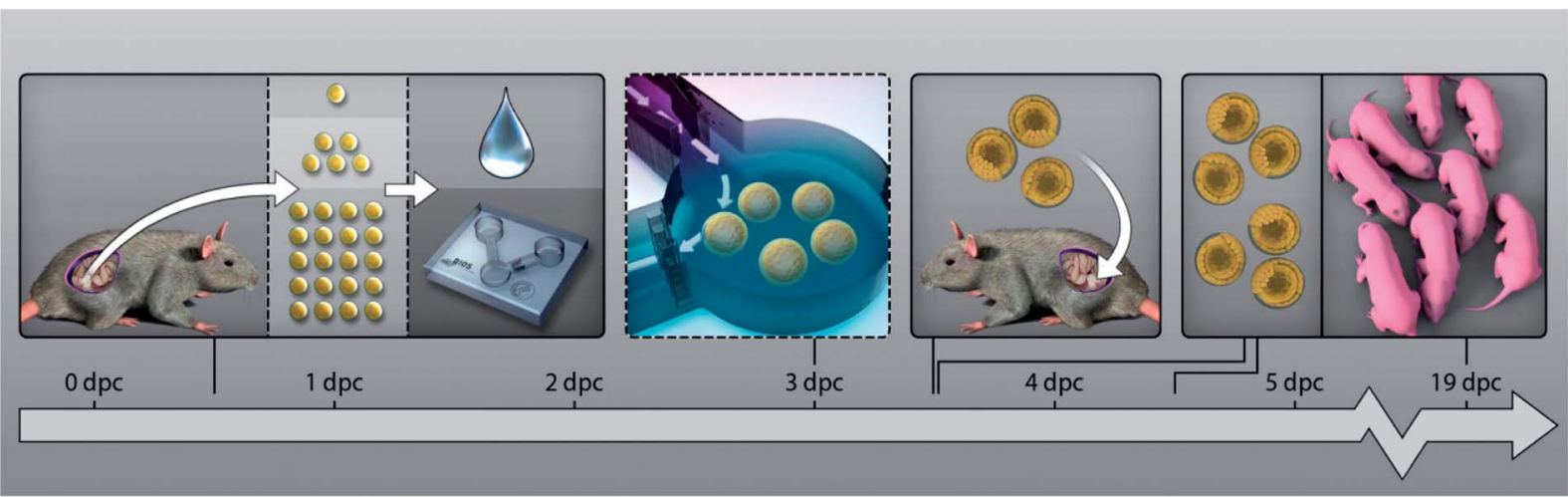

Fig. 1 Experimental design. Naturally fertilized mouse oocytes (B6C3F1xCD1) are collected from copulated mice after mating (18 h post-hCG), randomly allocated to various group cultures, and cultured in vitro from the zygote stage till the blastocyst stage (3.5-4.5 dpc) in $\alpha$-MEM medium. Culture groups are characterized by the embryo number (1, 5 or 20), the culture format employed for their in vitro development (microfluidic system or droplet), the volume employed for the culture (30 $\mathrm{nL}$, $270 \mathrm{~nL}$ or $5 \mu \mathrm{L}$ ), and by the implementation or not of a medium refreshment step at day 3 after fertilization. At $3.5 \mathrm{dpc}$, a subset of embryos is retrieved from the microfluidic chambers and droplets and transferred to the uterus of pseudo-pregnant mice to assess their full-term competence. Embryo growth is assessed at various time-points: during the pre-implantation period at $3.5 \mathrm{dpc}$ and $4.5 \mathrm{dpc}$, for their developmental stage; at $4.5 \mathrm{dpc}$, for the number of cells; and for their full-term development. Allocation of the embryos to the different experimental groups is detailed in Tables 1 and 2.

of experiments (groups of 5 embryos, microfluidics), medium is replenished at $3 \mathrm{dpc}$, as to determine whether medium exchange affects embryo development. Embryo developmental competence is assessed in terms of pre-implantation development (at 3.5 and $4.5 \mathrm{dpc}$ ) and cell count (at $4.5 \mathrm{dpc}$ ), as well as full-term development after embryo transfer in pseudo-pregnant recipient mice. The proposed microfluidic approach combined with single embryo culture opens novel possibilities for the field of developmental and reproductive biology, towards the identification of optimal in vitro culture conditions and the elucidation of the embryo's physiology.

\section{Materials and methods}

\section{Microsystem fabrication}

Microfluidic devices are fabricated in PDMS (polydimethylsiloxane) using standard soft lithography techniques. ${ }^{19}$ Briefly, a siliconbased mould is first fabricated in the cleanrooms of the MESA + Institute for Nanotechnology using photolithography and dryetching techniques, based on a design drawn with Clewin (WieWeb software, Hengelo, The Netherlands). This mould is subsequently coated with FDTS (perfluorodecyltrichlorosilane; ABCR, GmBH, Germany) to decrease the surface energy and facilitate the removal of the PDMS layer. Thereafter, a 10:1 PDMS pre-polymer:curing agent mixture (Sylgard 184, Dow Corning GmbH, Wiesbaden, Germany) is prepared and thoroughly degassed, poured on the mould, degassed once more and let for curing overnight at $60^{\circ} \mathrm{C}$. Following this, the PDMS layer is released from the mould, cut into individual devices using a sharp knife, and reservoirs are punched with sharp needles. Finally, the devices are bonded to glass cover slips using oxygen plasma treatment.

\section{Flow characterization and medium refreshment protocol}

The technique of passive pumping ${ }^{20}$ is employed for fluid manipulation in the device and medium refreshment. The flow profile and flow-rates are determined experimentally using $3 \mu \mathrm{m}$ diameter Polybead Polystyrene Violet Dyed beads (Polysciences Inc, Warrington, Pennsylvania, USA). Based on the passive pumping technique, a protocol for complete medium refreshment in the device is developed using fluorescent dyes (fluorescein sodium salt, and rhodamine B; both purchased from Sigma, Zwijndrecht, The Netherlands). The device is initially filled in with a fluorescein solution and droplets $(1.5 \mu \mathrm{L})$ of a rhodamine B solution are applied in the inlet reservoir and pumped in the device until no green fluorescence is detected anymore in the $\mathrm{nL}$ chamber. These characterization experiments are carried out in empty chambers and chambers loaded with $80 \mu \mathrm{m}$ microbeads (Megabead NIST Traceable Particle Size Standard, Polysciences Inc, Warrington, Pennsylvania USA), which are used as surrogates for mouse embryos.

\section{Animal handling}

Mice are maintained and handled at the animal facility of the Max-Planck-Institute in Münster, according to the breeding/ keeping licence and the ethical permit issued by the Landesamt für Natur, Umwelt und Verbraucherschutz (LANUV) of the state of North Rhine-Westphalia, Germany. Mice are housed in groups of five, exposed to $14 \mathrm{~L}: 10 \mathrm{D}$ (light : dark) hours photoperiod, and are fed ad libitum on Harlan-Teklad 2020SX diet (Harlan Laboratories, Oxford, United Kingdom). Five to seven week old females of the $\mathrm{B} 6 \mathrm{C} 3 \mathrm{~F} 1$ strain $(\mathrm{C} 57 \mathrm{Bl} / 6 \mathrm{~J} \times \mathrm{C} 3 \mathrm{H} / \mathrm{HeN})$ are superovulated by intra-peritoneal injection of 10 international units (IU) of pregnant mare's serum gonadotropin (PMSG; Intergonan, Intervet, Germany), followed by 10 IU of human chorionic gonadotropin (hCG; Ovogest, Intervet, Germany) $48 \mathrm{~h}$ later. Immediately after hCG injection, females are mated to CD1 stud males. Copulated females (identified the next morning by presence of a vaginal plug) are sacrificed after cervical dislocation $17 \mathrm{~h}$ after hCG injection, and 1-cell embryos (zygotes) are retrieved from the oviductal ampullae. Zygotes are handled in Hepes-buffered CZB (HCZB) medium, as previously described, ${ }^{21,22}$ and are separated from any remaining cumulus 
cells by treatment with $50 \mathrm{IU} \mathrm{mL}^{-1}$ hyaluronidase (ICN Biomedicals, Eschwege, Germany) in HCZB medium, followed by gentle pipetting.

\section{Culture of mouse embryos in microfluidic chambers and droplets}

Mouse embryo culture experiments are conducted over several days and using different batches of microfluidic systems (different experimental series), in order to assess biological and microdevice reproducibility. Microfluidic systems are washed with $70 \%$ ethanol, twice with fresh Milli Q water prior to use, and placed in $35 \mathrm{~mm}$ Petri-dishes (Sarstedt, Nümbrecht, Germany). 2 mL of alpha-modified Eagle's medium ${ }^{23,24}$ ( $\alpha$-MEM, M4526 Sigma, Germany) supplemented with $0.2 \% \mathrm{w} / \mathrm{v}$ BSA (Pentex; Serological Proteins Inc., Kankakee, IL, USA) and $50 \mu \mathrm{g}$ $\mathrm{mL}^{-1}$ gentamicin sulphate (ICN Biomedicals, Irvine, CA, USA) are finally added in the Petri dish around the microsystem to preserve a high level of humidity and prevent medium evaporation in the device. The reduced final medium volume is thereby kept below reservoir level, avoiding contact with the surface and interior of the microfluidic system. Devices are filled with the same culture medium and pre-incubated at $37{ }^{\circ} \mathrm{C}$ under $7 \% \mathrm{CO}_{2}$. Zygotes are placed in the inlet reservoir using a curved polished glass pipette operated through a mouthpiece, and subsequently transferred to the culture chamber using passive pumping. ${ }^{20}$ Embryos are cultured at $37{ }^{\circ} \mathrm{C}$ under $7 \%$ $\mathrm{CO}_{2}$ until further manipulation. For one set of experiments, medium is replenished in the microfluidic chambers ("dynamic culture"), as described in the previous section. For that purpose, medium is maintained at culture conditions $\left(37^{\circ} \mathrm{C}\right.$ under $7 \%$ $\mathrm{CO}_{2}$ ) prior to medium exchange. When outside the incubator, microfluidic systems are operated in a room at $30{ }^{\circ} \mathrm{C}$ as quickly as possible. Thereby, in terms of physical conditions, dynamic culture experiments follow the protocols used in conventional embryo culture as closely as possible. For control experiments, conditions used usually in embryology studies as well as ART culture are used: embryos are grown in $5 \mu \mathrm{L}$ droplets (in the same culture medium), placed in a sterile Petri-dish and covered with mineral oil suitable for mouse embryo culture (Sigma M8410), to prevent medium evaporation. Embryo development during culture in both microfluidic chambers and droplets is imaged using a stereomicroscope (Nikon SMZ800, Plan Apo 1X WD70, Düsseldorf, Germany) coupled to a camera (Nikon, DS-2Mv) and a computerized image acquisition system (ACT-2U Version 1.51.116.256).

\section{Embryo transfer}

Embryos are retrieved from microfluidic systems at $3.5 \mathrm{dpc}$ by applying $10 \mu \mathrm{L}$ of culture medium on the outlet reservoir to create a mild back-flow in the chamber. Thereby, the embryos are displaced through the V-shape structures into the inlet reservoir. There, embryos are collected using a flame-polished glass pipette and placed in $400 \mu \mathrm{L}$ of HCZB medium (Nunc 4-well dish; Thermo Fisher Scientific, Langenselbold, Germany), until further processing. Embryos are selected for embryo transfer (ET) on the basis of the presence of a cavity (blastocoel), which is indicative of the blastocyst stage; in a situation where embryos would progress in pre-implantation development at a slower rate (i.e., reaching at least the morula stage, not showing a cavity), morula embryos (no cavity) have also been used for ET (random sampling). Embryo transfer is performed as previously described..$^{21,22}$ Blastocysts are transferred in groups of 9-11 embryos to the left uterine horn of each pseudo-pregnant female (one experimental condition per female). Pregnancies are allowed to progress to term.

\section{Blastocyst cell count}

After retrieval, blastocysts are stained at $4.5 \mathrm{dpc}$ for cell count using nucleus staining (DRAQ5). Imaging is performed with an UltraVIEW RS 3ES spinning disk confocal imaging system (PerkinElmer LAS GmbH, Jügesheim, Germany) on an inverted microscope (TE-2000U, Nikon GmbH, Düsseldorf, Germany) using a 60x water immersion lens (N.A. 1.2). Acquired images are imported into Volocity (PerkinElmer, Waltham, MA, USA) and processed for cell counting (based on nuclear staining) using maximum intensity projections.

\section{Statistical analysis}

Blastocyst and birth rates (i.e., proportions) are analyzed according to Norman TJ Bailey (Statistical methods in biology, 1995, Cambridge University Press). Briefly, given two groups, one of $n_{1}$ embryos with $a_{1}$ having a certain character (e.g., blastocyst formation), the other of $n_{2}$ embryos with $a_{2}$ having the character, the mathematical formula of Bailey answers the question of whether the two groups may be considered as drawn from the same population. First, the following parameters are calculated: $k_{1}=\frac{a_{1}}{n_{1}} ; \quad k_{2}=\frac{a_{2}}{n_{2}} ; k=\frac{a_{1}+a_{2}}{n_{1}+n_{2}}$.

Subsequently, $d$ the units of standard deviation that separate the two groups from each other is determined: $d=\frac{k_{1}-k_{2}}{\sqrt{\left(k \times(1-k) \times\left(\frac{1}{n_{1}}+\frac{1}{n_{2}}\right)\right)}} \cdot d$ is referred to the areas of the normal curve. Values of $d$ higher than 1.960 will cause to reject the null hypothesis at a confidence level of 5\% (two tails).

\section{Results}

\section{Microfluidic environment for embryo pre-implantation culture}

To more closely recapitulate the physical confinement found in the mouse oviduct, a microfluidic system is developed that includes a culture chamber with nanoliter capacity (30 or 270 nL; see Fig. 2). Two reservoirs are connected to the chamber via microchannels for the introduction of solutions and embryos. Importantly, this configuration allows diffusion-based delivery of nutrients from the reservoirs of the device as well as accumulation of factors secreted by the embryos. ${ }^{25}$ Altogether, gradients are established during the culture in the vicinity of the embryo(s), mimicking thereby conditions found in vivo. Furthermore, structures are added at the inlet and outlet of the chamber to trap embryo(s) during the culture while allowing 


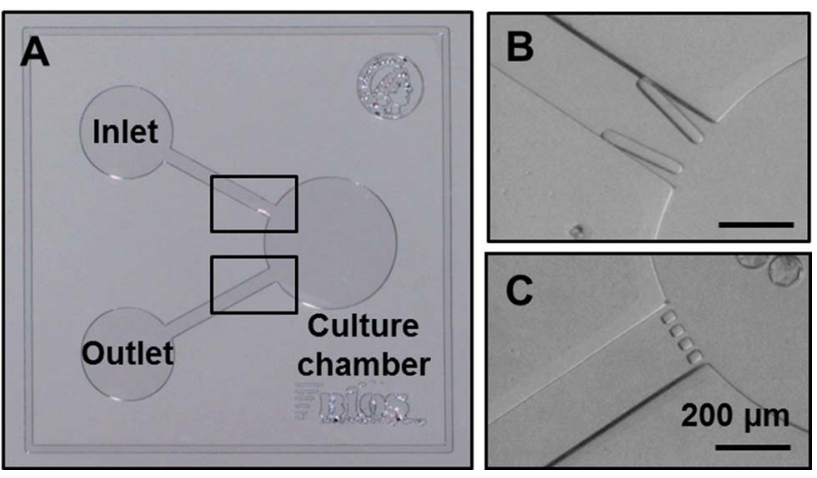

Fig. 2 Microfluidic device for embryo culture. Picture of the device fabricated from PDMS prior to bonding on a glass substrate: the device includes a $\mathrm{nL}$ volume chamber in which embryos are cultured. Embryos are introduced from the inlet reservoir, guided in the chamber ( $V$-shaped structure; $70 \mu \mathrm{m}$ spacing; top right) and trapped therein with the help of grids ( $20 \mu \mathrm{m}$ spacing; bottom right). All scale bars represent $200 \mu \mathrm{m}$.

their retrieval (Fig. 2). The possibility to recover the embryos from microfluidic devices has been previously deemed essential in order to test for full-term development, ${ }^{2}$ but has not yet been demonstrated. The V-shaped structures implemented at the inlet of the chamber present a $70 \mu \mathrm{m}$ spacing, which is slightly smaller than the size of mouse embryos $(c a .80 \mu \mathrm{m})$ to enable their passage when a mild flow is applied, while preventing them from moving out of the chamber in the absence of flow. All microfluidic structures are fabricated from polydimethylsiloxane (PDMS), which has been validated for its inertness towards embryos (ESI SI-1†), and bonded to a glass substrate.

\section{Flow characterization}

Determination of flow settings inside the microfluidic chamber is essential to characterize culture conditions in the proposed device. Flows are created in the device using "passive pumping", ${ }^{20}$ which relies on the deposition of round droplets of different sizes on the inlet and outlet reservoirs, without the need for complex fluidic connections. Flows in the chamber are characterized as a function of the chamber dimensions with the help of $3 \mu \mathrm{m}$ beads added in the liquid. Fig. 3A illustrates the flow profile observed in a $270 \mathrm{~nL}$ chamber when a $1.5 \mu \mathrm{L}$ droplet is applied in the inlet and a $10 \mu \mathrm{L}$ droplet on the outlet. A flow gradient is found along the outlet grids, where embryos are generally located. The maximal flow value is estimated to be around $64.8 \mu \mathrm{L} \mathrm{h}^{-1}$ in absence of embryos, decreasing when embryos are present in the chamber (embryos reduce flow as they occupy the chamber close to the outer microchannel; e.g., $50 \%$ reduction for 20 embryos in a $270 \mathrm{~nL}$ chamber).

Once the flow settings have been characterized, the number of pumping cycles (using $1.5 \mu \mathrm{L}$ droplets) required to fully refresh the content of the chamber is determined using fluorescent dyes (Fig. 3B): a rhodamine solution is pumped in a chamber previously filled with a fluorescein solution (Fig. 3B). Typically, 3 or 7 cycles are required to completely replace the culture medium in $30 \mathrm{~nL}$ and $270 \mathrm{~nL}$ chambers, respectively, as indicated in Fig. 3C. The presence of embryo(s) in the culture chamber is found to have a negligible influence on this medium refreshment protocol (data not shown).

\section{Pre-implantation development characterization in microfluidic environment and conventional droplets}

In mouse development, the physiological time window for blastocyst formation (i.e., the embryonic stage in preparation for implantation in the uterus) ranges from 3.5 to $4.5 \mathrm{dpc}$ in vivo, with cavity (blastocoel) formation starting at $72 \mathrm{~h}$ post fertilization. Mouse embryos cultured in the microfluidic systems develop significantly faster than in droplets, as measured by the earlier appearance of a cavity and its larger size at $3.5 \mathrm{dpc}$ (Table 1). This result is independent of the chamber volume and applies to embryos cultured as groups of 5 , or individually. For pools of 20 embryos, the chamber volume impacts on the development rate: blastocyst formation at $3.5 \mathrm{dpc}$ is delayed for embryos cultured in $30 \mathrm{~nL}$ chambers (only $13.3 \%$ blastocysts), while in $270 \mathrm{~nL}$ chambers the blastocyst rate $(54.7 \%)$ is only slightly lower than for smaller embryo groups. Interestingly, in droplets, none of the embryos cultured individually reaches the blastocyst stage at $3.5 \mathrm{dpc}$, while $72.3 \%$ and $63.1 \%$ of those placed in 30 and $270 \mathrm{~nL}$ chambers, respectively, already present a cavity (see Fig. 4).

At $4.5 \mathrm{dpc}$, the microfluidic environment significantly increases the chance of mouse embryos to become blastocysts compared to droplets (Table 1). All microfluidics conditions yield blastocyst rates above $90 \%$, compared to significantly lower rates in drops $(29.8,66.6$ and $73.4 \%$ for 1,5 , and 20 embryos, respectively). The results concerning drop culture of mouse embryos are in full agreement with previously published data. For instance, Kato and Tsunoda reported blastocyst rates of 38 and $63 \%$ for the culture of single embryos and pools of 10 embryos, respectively, in $10 \mu \mathrm{L}$ microdrops under similar conditions. $^{26}$ Furthermore, the development of embryos cultured as pools of 20 in $30 \mathrm{~nL}$ chambers is restored at $4.5 \mathrm{dpc}$ $(90.8 \%)$ with rates comparable to all microfluidic conditions. The widely reported group size effect in pre-implantation development, ${ }^{26-29}$ which is also found here in the droplet culture, is not observed in the microfluidic environment.

As previously reported for various mammalian species, the total cell number is considered as a good indicator for the quality of pre-implantation embryos, as well as a predictor for good development, ${ }^{30-33}$ with morphological blastocyst scoring methods correlating with cell numbers in the human. ${ }^{34}$ In the present study, within the same experiment, single embryo culture produces a significant increase in total blastocyst cell numbers at $4.5 \mathrm{dpc}$, in both $30 \mathrm{~nL}(78 \pm 23$ cells, $n=7 ; p=$ $0.0002)$ and $270 \mathrm{~nL}$ ( $54 \pm 10$ cells, $n=5 ; p=0.009)$ chambers compared to droplets ( $34 \pm 7$ cells, $n=10$ ). Most importantly, total cell counts for single embryo culture in a microfluidic environment are comparable to those of embryos cultured in pools of 20 in droplets ( $30 \mathrm{~nL} p=0.57 ; 270 \mathrm{~nL} p=0.05$ ). Also in droplets, total cell numbers are significantly higher for embryos cultured in pools of 20 (72 \pm 13 cells, $n=8)$ than for single culture ( $34 \pm 7$ cells, $n=10 ; p=6.4 \times 10^{-6}$ ), as already widely reported in the literature. ${ }^{26,35}$ 

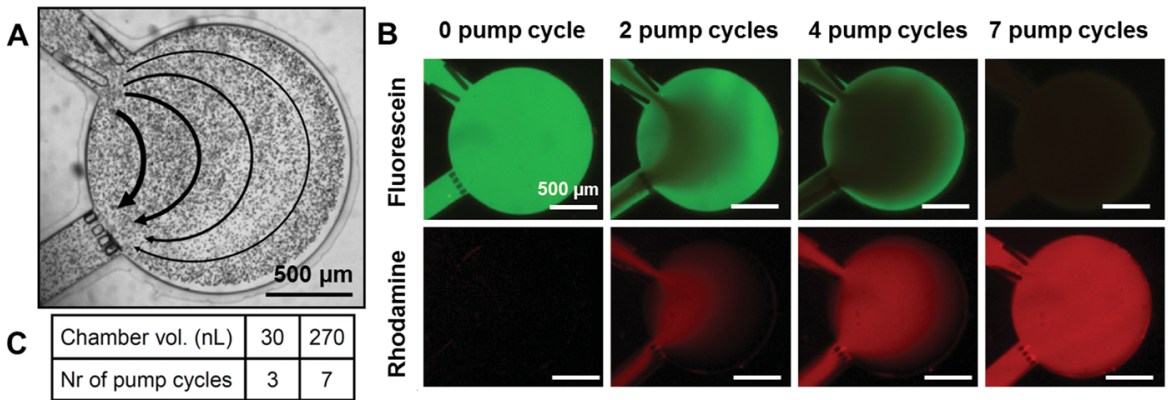

Fig. 3 Fluidic characterization of the microfluidic device. (A) Flow profile in a $270 \mathrm{~nL}$ chamber using passive pumping ${ }^{20}$ after supplementation of the liquid with microbeads (pumping droplet of $1.5 \mu \mathrm{L}$ ). Flow values from right to left: $3.9 \mu \mathrm{L} \mathrm{h}^{-1}, 6.5 \mu \mathrm{L} \mathrm{h}^{-1}, 16.2 \mu \mathrm{L} \mathrm{h}^{-1}, 32.4 \mu \mathrm{L} \mathrm{h}^{-1}$. (B and C) Development of a medium refreshment protocol. The device is firstly filled with a solution of fluorescein in PBS (green), and a rhodamine B solution (red) in PBS is pumped using the passive pumping technique in the chamber using $1.5 \mu \mathrm{L}$ droplets $(1.5 \mu \mathrm{L}=1$ pumping cycle) until no green fluorescence is detected. (B) Pictures showing the process for a $270 \mathrm{~nL}$ chamber after 0,2 , 4, and 7 pumping cycles. All scale bars represent $500 \mu \mathrm{m}$. (C) Table showing the number of pumping cycles necessary to fully refresh $30 \mathrm{and} 270 \mathrm{~nL}$ chambers.

\section{Full-term development}

Mouse embryo culture has already been reported using microdevices. ${ }^{12-15,17,18}$ Most of these studies concur in indicating that a confined environment as found in microfluidic systems gives developmental advantage during early pre-implantation, compared to the conventional droplet approach. ${ }^{\mathbf{1 3 , 1 4}}$ However, the demonstration of pre-implantation development alone is not sufficient to prove that an in vitro culture system does not harm the embryos. ${ }^{22}$ Therefore, we examine the full-term development of mouse embryos cultured throughout the preimplantation period in a confined $\mathrm{nL}$ volume chamber.

Single embryo culture in droplets supports lower birth rates (20\%) compared to group culture $(37.5 \%)$; these data are in good agreement with previously published reports..$^{26,28,35}$ On the contrary, full-term development of individually cultured mouse embryos is enhanced in a microfluidic confined environment (30 nL: $33.3 \% ; 270 \mathrm{~nL}: 28.9 \%$ ) compared to drops $(20 \%)$. Furthermore, for culture in microfluidic devices, the chamber capacity (30 and $270 \mathrm{~nL}$ ) has no influence on the embryo developmental competence, while the embryo number does. Groups of 20 embryos in chambers give the highest birth rates (30 nL: 42.8\%; 270 nL: 45.4\%) surpassing standard (i.e., droplet) culture conditions $(37.5 \%)$, while surprisingly for groups of 5 embryos the lowest birth rates are observed (30 nL: 19.1\%; $270 \mathrm{~nL}: 15.2 \%)$. These unexpected variations in the developmental rates as a function of the group size raise interesting biological questions for future studies. A possibility is that a fine balance exists between the amount of essential (e.g., growth factors) and deleterious molecules released by the embryos themselves, as well as the availability of the right physical conditions (such as availability of nutrients and oxygen).

Most importantly, our data show that mouse embryos can develop in a microfluidic environment under the prescriptions of human ART, i.e., single embryo culture. So far, various attempts to culture single mouse embryos in droplets have led to impaired development and a decrease in cell numbers, ${ }^{26,35}$

Table 1 Pre-implantation development of mouse embryos in a microfluidic environment and conventional drops. Pre-implantation development of mouse embryos in $\mathrm{nL}$ microfluidic chambers ( 30 and $270 \mathrm{~nL}$ ) and conventional drops $(5 \mu \mathrm{L}$ ), characterized in terms of blastocyst number (blast. nr.) obtained at 3.5 and 4.5 days post coitum $(\mathrm{dpc})$, relative to starting number of embryos in culture (starting embryo $\mathrm{nr}$.). A recount of the starting embryo nr. is done at $2 \mathrm{dpc}$, not to include those that did not progressed to 2 -cell embryos (likely to be unfertilized oocytes). Culture conditions tested include format ( $\mathrm{nL} v \mathrm{vs}, \mu \mathrm{L})$, culture volume $(30,270 \mathrm{~nL} v \mathrm{~s}$. $5 \mu \mathrm{L})$, embryo $\mathrm{nr}$. (1, 5 or 20) and flow conditions (static vs. dynamic, dyn.)

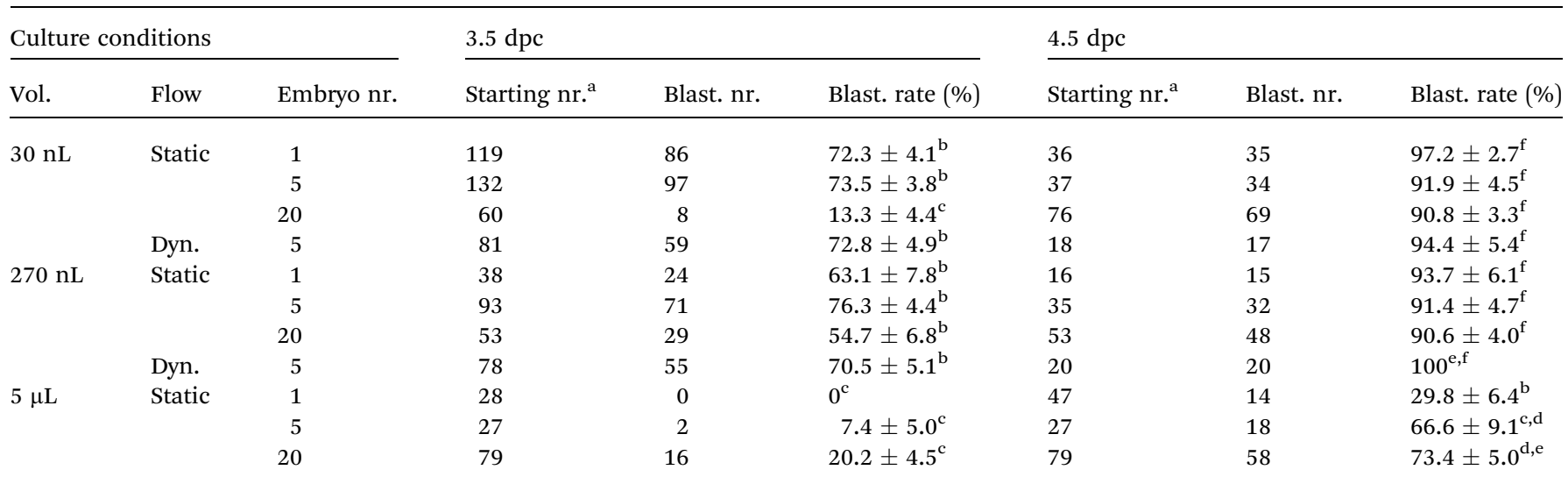

${ }^{\mathrm{a}}$ Unfertilized embryos (i.e., one-cell at $2.5 \mathrm{dpc}$ ) have been removed from the calculations, ${ }^{\mathrm{b}, \mathrm{c}, \mathrm{d}, \mathrm{e}, \mathrm{f}}$ values sharing one superscript letter are not significantly different. 
A. Static culture, 5 embryos, 30-nL chamber

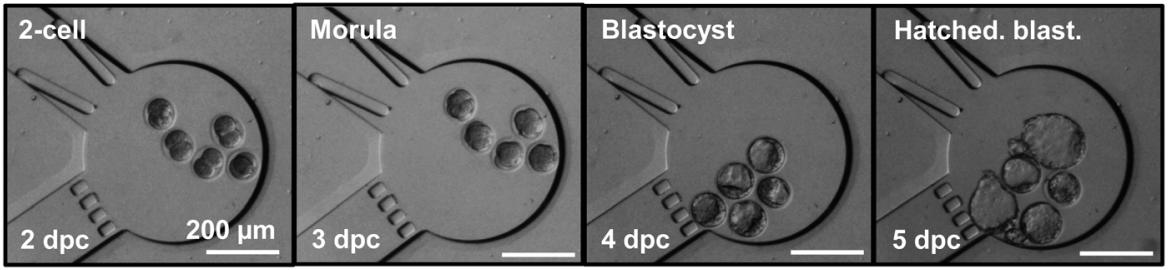

B. Dynamic culture

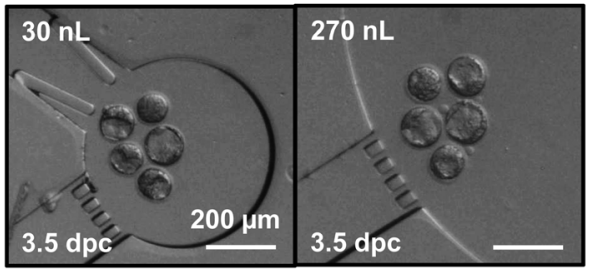

\section{Static culture}

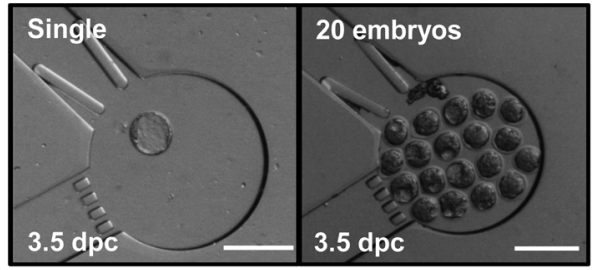

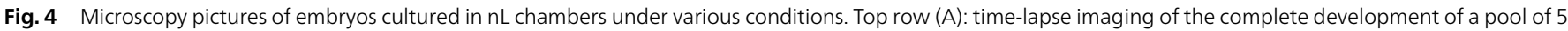

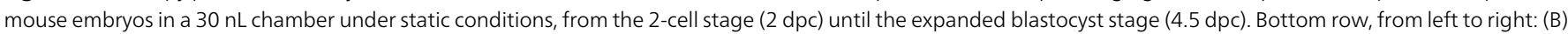

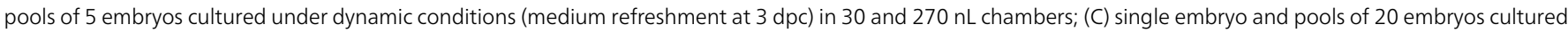
under static conditions in $30 \mathrm{~nL}$ chambers. All pictures in (B) and (C) are taken at $3.5 \mathrm{dpc}$. All scale bars represent $200 \mu \mathrm{m}$.

both being restored upon supplementation of specific growth factors in the medium. ${ }^{28}$ In the present study, we demonstrate for the first time viable full-term development of embryos cultured individually in a confined microfluidic environment, with growth rates and cell numbers comparable to embryos cultured in groups in either microfluidic chambers or conventional droplets (Table 2). The confinement found in the chambers is likely to play a crucial role for single embryo development, namely through the local accumulation of growth

Table 2 Full-term development of mouse embryos in a microfluidic environment and conventional drops. Full-term development of mouse embryos after pre-implantation culture in a $\mathrm{nL}$ microfluidic chamber (30 and $270 \mathrm{~nL}$ ) and conventional drops $(5 \mu \mathrm{L})$, characterized in terms of pups born with respect to the number of $3.5 \mathrm{dpc}$ blastocysts transferred (embryo nr.; 9-11 embryos were allocated per female). Birth rate is defined as percentage of live young/transferred blastocysts in those females that became pregnant. Culture conditions tested include format ( $\mathrm{nL} v$ s. $\mu \mathrm{L}$ ), culture volume (30, $270 \mathrm{~nL}$ vs. $5 \mu \mathrm{L})$, embryo number (1, 5 or 20 ) and flow conditions (static, stat. vs. dynamic, dyn.)

\begin{tabular}{|c|c|c|c|c|c|}
\hline \multicolumn{3}{|c|}{ Culture conditions } & \multicolumn{3}{|l|}{$3.5 \mathrm{dpc}$} \\
\hline Vol. & Flow & Embryo nr. & Starting nr. & Pups nr. & Birth rate (\%) \\
\hline \multirow[t]{4}{*}{$30 \mathrm{~nL}$} & Static & 1 & 36 & 12 & $33.3 \pm 7.9^{\mathrm{c}, \mathrm{d}}$ \\
\hline & & 5 & 47 & 9 & $19.1 \pm 5.7^{\mathrm{a}, \mathrm{d}}$ \\
\hline & & 20 & 28 & 12 & $42.8 \pm 9.4^{\mathrm{c}}$ \\
\hline & Dyn. & 5 & 39 & 11 & $28.2 \pm 7.2^{\mathrm{a}, \mathrm{c}, \mathrm{d}}$ \\
\hline \multirow[t]{4}{*}{$270 \mathrm{~nL}$} & Static & 1 & 45 & 13 & $28.9 \pm 6.8^{\mathrm{a}, \mathrm{c}, \mathrm{d}}$ \\
\hline & & 5 & 59 & 9 & $15.2 \pm 4.7^{\mathrm{d}}$ \\
\hline & & 20 & 22 & 10 & $45.4 \pm 10.6^{\mathrm{b}, \mathrm{c}}$ \\
\hline & Dyn. & 5 & 34 & 10 & $29.4 \pm 7.8^{\mathrm{b}, \mathrm{d}}$ \\
\hline \multirow[t]{3}{*}{$5 \mu \mathrm{L}$} & Static & 1 & 20 & 4 & $20.0 \pm 8.9^{\mathrm{b}, \mathrm{d}}$ \\
\hline & & 5 & 40 & 15 & $37.5 \pm 7.7^{\mathrm{a}, \mathrm{b}, \mathrm{c}}$ \\
\hline & & 20 & 40 & 15 & $37.5 \pm 7.7^{\mathrm{a}, \mathrm{b}, \mathrm{c}}$ \\
\hline \multirow[t]{2}{*}{$30+270 \mathrm{~nL}$} & Static & 5 & 106 & 18 & $16.9 \pm 3.6^{\mathrm{a}}$ \\
\hline & Dyn. & & 73 & 21 & $28.8 \pm 5.3^{b}$ \\
\hline $5 \mu \mathrm{L}$ & Static & 5 & 40 & 15 & $37.5 \pm 7.7^{\mathrm{b}}$ \\
\hline
\end{tabular}

a,b,c,d Values sharing one superscript letter are not significantly different. factors secreted by the embryos ${ }^{25}$ that promote their development ${ }^{28}$ and the formation of gradients of nutrients in the embryo environment, as found in vivo., ${ }^{\mathbf{1 , 3 6}}$ The microfluidic system presented here provides a valuable platform for the future clarification of all the above hypotheses.

\section{Medium perfusion and embryo developmental competence}

Medium refreshment during culture is generally considered to promote embryo development. ${ }^{\mathbf{1 6}}$ Medium substitution is also a common procedure in many ART laboratories, as different media compositions are believed to bring specific beneficial effects at different pre-implantation stages. In other cases, medium is simply refreshed during the culture. In our study, this parameter is tested on groups of 5 embryos in microfluidic chambers. Refreshing the medium at $3 \mathrm{dpc}$ (as for ART sequential media protocols) has no influence on embryo preimplantation rates (Table 1), most probably as the medium is replenished only shortly before blastocyst rates are assessed (3.5 dpc). Interestingly, medium refreshment promotes fullterm development over static conditions (Table 2), as shown by the significant increment in birth rates (from $16.9 \%$ to $28.8 \%$ for microfluidic systems without and with medium refreshment, respectively).

Hickman et al. showed impaired development for embryos exposed to a continuous but low flow-rate $\left(0.1-0.5 \mu \mathrm{L} \mathrm{h}^{-1}\right),{ }^{12}$ and a 1.2 dyne $\mathrm{cm}^{-2}$ shear stress has been reported as lethal for mouse embryos. ${ }^{37}$ On the contrary, pulsatile delivery of medium has recently been demonstrated to promote embryo development. ${ }^{16}$ Here, embryo development seems to benefit from the transient (1-2 min) but much higher flow (4-50 $\mu \mathrm{L} \mathrm{h}^{-1}$; maximum shear stress ca. 0.17 dyne $\mathrm{cm}^{-2}$ ) which is created upon punctual medium refreshment using passive pumping. ${ }^{20}$ We hypothesize this positive impact originates from the mild and punctual mechanical stimulation exerted on the embryos. Interestingly, this emulates the conditions the embryos 
experience in vivo throughout their first days of development, particularly when they travel in the oviduct from the ovary to the uterus. ${ }^{16}$

\section{Conclusion}

This study is the first comprehensive account that a nanoliter volume not only can support pre-implantation development in vitro, but it can also preserve the post-implantation ability, resulting in full-term development after blastocyst transfer to the uterus. The system offers the possibility to non-invasively assess the impact of culture conditions on embryo growth. In particular, this set of experiments has revealed three notable features: first, microfluidic chambers support full-term development of mouse embryos with birth rates comparable to droplet culture; second, medium refreshment in microfluidic systems (tested here on groups of 5 embryos) has a significant impact on embryo full-term development; third, and most importantly, single embryo culture in a microfluidic environment gives birth rates and cell numbers comparable to group culture in droplets. This last key result - that mouse embryos develop further to term when cultured in a confined environment under the prescription of human ART, i.e., single embryo culture - is a major step for the field of ART. The reason why post-implantation rates are here - in the mouse - varying across group sizes while being independent of the microfluidic volume remains to be investigated. However, it is known that mouse blastocyst rates are not predictive of foetal rates, whereas blastocyst cell numbers are. ${ }^{32}$ The most parsimonious explanation for the lack of difference in full development is that the minimum viable number of cells was secured in all of our experiments owing to the fact that mouse oocytes were fertilized in vivo, i.e., under best conditions. It will be interesting also to see the effect of microfluidic embryo culture on embryos fertilized by other ART methods such as intracytoplasmic sperm injection (ICSI), which is an invasive technique predicted to affect blastocyst cell numbers. Considering the historical records in which single embryo culture - which is praxis in human ART - has always proven to be problematic for the mouse, ${ }^{26-28}$ our present achievements narrow the gap between the mouse as a model for human embryo development and human ART, by establishing that mouse embryos can be cultured in a way that matches human embryos (i.e., at the single embryo level). In parallel, the milestone achieved in this study also enables re-thinking embryo culture in microfluidic platforms comprising individual chambers for parallel single embryo culture. This single embryo approach suppresses risks of low quality embryos negatively influencing nearby fellow embryos, ${ }^{38}$ and facilitates embryo tracking during the culture while enabling to tailor culture conditions for each embryo. Importantly, physiological data can be collected at the single embryo level using dedicated sensors and analytical tools implemented in $\mathrm{nL}$ chambers or microdevices. ${ }^{39-43}$ Such data will undoubtedly not only help identifying the physiological requirements of pre-implantation embryos, but also provide new means to monitor embryo growth and assess their quality using more rational criteria than morphology alone, ${ }^{44}$ as currently done in the clinics. Furthermore, embryo development can simultaneously be followed using time-lapse imaging $^{45}$ in the microfluidic chambers, e.g., to correlate physiological data and developmental stage. Finally, this combination of single embryo culture in microfluidic systems with in situ characterization scheme is highly valuable to screen physical and chemical culture parameters, as undertaken in the present study, towards the determination of an optimal in vitro culture protocol.

\section{Acknowledgements}

The authors would like to thank the technicians of the BIOS group for their precious help, as well as the animal caretakers at the MPI Münster, for animal work. This research is funded by the bilateral project NWO-DFG DN 63-258 and the DFG BO 2540/4-1 (MB).

\section{References}

1 G. Vajta, L. Rienzi, A. Cobo and J. Yovich, Reprod. BioMed. Online, 2010, 20, 453-469.

2 J. E. Swain and G. D. Smith, Hum. Reprod. Update, 2011, 17, 541-557.

3 G. A. Thouas, G. M. Jones and A. O. Trounson, Reproduction, 2003, 126, 161-169.

4 G. Vajta, T. Korosi, Y. Du, K. Nakata, S. Ieda, M. Kuwayama and Z. P. Nagy, Reprod. BioMed. Online, 2008, 17, 73-81.

5 G. Vajta, T. T. Peura, P. Holm, K. Paldi, T. Greve, A. O. Trounson and H. Callesen, Mol. Reprod. Dev., 2000, 55, 256-264.

6 G. M. Whitesides, Nature, 2006, 442, 368-373.

7 T. M. Keenan and A. Folch, Lab Chip, 2008, 8, 34-57.

8 S. Le Gac and A. van den Berg, Trends Biotechnol., 2010, 28, 55-62.

9 E. M. Lucchetta, J. H. Lee, L. A. Fu, N. H. Patel and R. F. Ismagilov, Nature, 2005, 434, 1134-1138.

$10 \mathrm{H}$. Hwang and H. Lu, Biotechnol. J., 2013, 8, 192-205.

11 T. J. Levario, M. Zhan, B. Lim, S. Y. Shvartsman and H. Lu, Nat. Protoc., 2013, 8, 721-736.

12 D. L. Hickman, D. J. Beebe, S. L. Rodriguez-Zas and M. B. Wheeler, Comp. Med., 2002, 52, 122-126.

13 S. Raty, E. M. Walters, J. Davis, H. Zeringue, D. J. Beebe, S. L. Rodriguez-Zas and M. B. Wheeler, Lab Chip, 2004, 4, 186-190.

14 J. Melin, A. Lee, K. Foygel, D. E. Leong, S. R. Quake and M. W. M. Yao, Dev. Dyn., 2009, 238, 950-955.

15 R. Ma, L. Xie, C. Han, K. Su, T. Qiu, L. Wang, G. Huang, W. Xing, J. Qao, J. Wang and J. Cheng, Anal. Chem., 2011, 83, 2964-2970.

16 Y. S. Heo, L. M. Cabrera, C. L. Bormann, C. T. Shah, S. Takayama and G. D. Smith, Hum. Reprod., 2010, 25, 613622.

17 C. Han, Q. Zhang, R. Ma, L. Xie, T. Qiu, L. Wang, K. Mitchelson, J. Wang, G. Huang, J. Qiao and J. Cheng, Lab Chip, 2010, 10, 2848-2854. 
18 M. S. Kim, C. Y. Bae, G. Wee, Y.-M. Han and J.-K. Park, Electrophoresis, 2009, 30, 3276-3282.

19 X. M. Zhao, Y. N. Xia and G. M. Whitesides, J. Mater. Chem., 1997, 7, 1069-1074.

20 G. M. Walker and D. J. Beebe, Lab Chip, 2002, 2, 131-134.

21 F. M. Cavaleri, S. T. Balbach, L. Gentile, A. Jauch, B. BbhmSteuer, Y.-M. Han, H. R. Schbler and M. Boiani, Mech. Dev., 2008, 125, 153-166.

22 C. Schwarzer, T. C. Esteves, M. J. Arauzo-Bravo, S. Le Gac, V. Nordhoff, S. Schlatt and M. Boiani, Hum. Reprod., 2012, 27, 2627-2640.

23 N. Desai, D. Kinzer, A. Loeb and J. Goldfarb, Hum. Reprod., 1997, 12, 328-335.

24 W. S. A. Wun, C. C. C. Wun and G. M. Grunert, J. Assisted Reprod. Genet., 1994, 11, 303-307.

25 H. M. Yu, C. M. Alexander and D. J. Beebe, Lab Chip, 2007, 7, 726-730.

26 Y. Kato and Y. Tsunoda, Theriogenology, 1994, 41, 1315-1322.

27 R. S. Canseco, A. E. T. Sparks, R. E. Pearson and F. C. Gwazdauskas, J. Assisted Reprod. Genet., 1992, 9, 454-457.

28 B. C. Paria and S. K. Dey, Proc. Natl. Acad. Sci. U. S. A., 1990, 87, 4756-4760.

29 M. Lane and D. K. Gardner, Hum. Reprod., 1992, 7, 558-562. 30 T. P. Fleming, Dev. Biol., 1987, 119, 520-531.

31 V. E. Papaioannou and K. M. Ebert, Development, 1988, 102, 793-803.

32 M. Lane and D. K. Gardner, J. Reprod. Fertil., 1997, 109, 153-164.
33 S. Iwasaki, N. Yoshiba, H. Ushijima, S. Watanabe and T. Nakahara, J. Reprod. Fertil., 1990, 90, 279-284.

34 K. Matsuura, N. Hayashi, C. Takiue, R. Hirata, T. Habara and K. Naruse, Fertil. Steril., 2010, 94, 1135-1137.

35 B. D. Bavister, Theriogenology, 1988, 29, 143-154.

36 B. D. Bavister, Hum. Reprod. Update, 1995, 1, 91-148.

37 Y. F. Xie, F. F. Wang, W. J. Zhong, E. Puscheck, H. L. Shen and D. A. Rappolee, Biol. Reprod., 2006, 75, 45-55.

38 R. E. Spindler and D. E. Wildt, Biol. Reprod., 2002, 66, 167173.

39 Y. S. Heo, L. M. Cabrera, C. L. Bormann, G. D. Smith and S. Takayama, Lab Chip, 2012, 12, 2240-2246.

40 J. P. Urbanski, M. T. Johnson, D. D. Craig, D. L. Potter, D. K. Gardner and T. Thorsen, Anal. Chem., 2008, 80, 65006507.

41 K. Komori, S. Fujii, K. Montagne, H. Nakamura, H. Kimura, K. Otake, T. Fujii and Y. Sakai, Sens. Actuators, B, 2012, 162, 278-283.

42 C. C. Wu, T. Saito, T. Yasukawa, H. Shiku, H. Abe, H. Hoshi and T. Matsue, Sens. Actuators, B, 2007, 125, 680-687.

43 C. O'Donovan, E. Twomey, J. Alderman, T. Moore and D. Papkovsky, Lab Chip, 2006, 6, 1438-1444.

44 A. T. Kjellberg, P. Carlsson and C. Bergh, Hum. Reprod., 2006, 21, 210-216.

45 C. C. Wong, K. E. Loewke, N. L. Bossert, B. Behr, C. J. De Jonge, T. M. Baer and R. A. R. Pera, Nat. Biotechnol., 2010, 28, 1115-U1199. 